\title{
Modos de conocimiento en Plotino
}

\author{
María Isabel SAnTa CRUZ \\ Universidad de Buenos Aires ${ }^{1}$ \\ Argentina
}

\begin{abstract}
Resumo. Modos de conhecimento em Plotino. Após recapitular as grandes linhas do sistema plotiniano, o estudo analisa três formas de conhecimento que caracterizam os três níveis do real ou hipostases. Na inteligência, noûs, chamada nível noético, há um tipo de conhecimento autêntico, que é o conhecimento de si. Na alma, psyché, nível dianoético, há um tipo de conhecimento discursivo, que é conhecimento de si apenas em um sentido secundário e derivado. No Um, tò hén, nível supranoético, só podemos falar em conhecimento de si em sentido translativo e « elevado", porque o primeiro princípio do real, por ser unidade absoluta, não admite o pensamento, nem de oura coisa nem de si mesmo, mas, ao mesmo tempo, não é algo inerte, mas plenitude absoluta. $\mathrm{O}$ estudo procura mostrar de que maneira Plotino se embasa no modo de conhecimento da inteligência, da qual se serve como ponto nodal, com o intuito de articular os três modos de conhecimento e de caracterizar um « infra-conhecimento de si », o da alma, e um « supra-conhecimento de si », o do Um.
\end{abstract}

Palavras-Chave. Plotino; modos de conhecimento; Um; inteligência; alma; conhecimento de si.

\section{Introducción}

La influencia de Plotino ha sido probablemente tan profunda como la de Platón o la de Aristóteles. Pese a ello, los filósofos - o algunos pretendidos filósofos - del siglo XX y XXI, que hacen uso, más de una vez desaprensivo y acrítico, de Platón y de Aristóteles, poco o nada se ocupan de él. Por cierto, un conocimiento más o menos preciso de las Enéadas parece ser patrimonio de especialistas en estudios clásicos y, en particular de quienes se han interesado por el pensamiento griego tardío. Ello se debe, probablemente, a múltiples razones de diferente índole. Entre ellas está el hecho de que el pensamiento de Plotino es difícil y sus escritos, en muchos puntos oscuros, presentan las ideas de modo totalmente asistemático. Como afirma Lloyd Gerson, no sin razón, "Las Enéadas están escritas en un estilo altamente comprimido, a

\footnotetext{
${ }^{1}$ Consejo Nacional de Investigaciones Científicas y Técnicas (CONICET).
} 
veces oscuro, y en ellas dialéctica, exégesis, alegoría y argumento técnico a menudo se entrelazan de modo perturbador. Se trata de una obra exótica, tanto en forma como en contenido"2. Por lo demás, los no especialistas, muchos de los cuales lo descartan a priori, pueden tener una visión simplista y estereotipada de Plotino, al que suelen considerar como un pensador ecléctico y/o dogmático, que no ofrece una adecuada argumentación. Tales juicios pueden tener como base el conocimiento de algunos textos o pasajes expresivos o de tono "entusiasta", que recurren a metáforas o alegorías, pero quienes emiten tales juicios muestran desconocimiento de las largas y complejas exposiciones especulativas y abstractas, que ocupan mucha mayor extensión y que son, por lo menos, igualmente importantes y probablemente más importantes ${ }^{3}$. Piénsese, para poner sólo un ejemplo, en los extensos y difíciles tratados VI 1, VI 2 y VI 3, sobre los géneros del ser. Desconocen también que una lectura comprensiva de las Enéadas requiere tener en cuenta cómo enfrenta Plotino a sus predecesores, especialmente a Platón, cómo los lee y cuál es la naturaleza del método filosófico exegético que aplica ${ }^{4}$.

Tras estas breves observaciones preliminares - que han rozado problemas que no abordaré aquí y que serían dignos de un extenso tratamiento - entraré en el tema que he elegido para este trabajo: la caracterización plotiniana de diferentes modos de conocimiento. Mi propósito es a la vez ambicioso y modesto. Ambicioso, porque toca un aspecto crucial en la filosofía de Plotino. Modesto, porque, tras recordar brevemente las líneas generales del pensamiento plotiniano, me limitaré a examinar un conjunto acotado de textos de las Enéadas, establecer algunas comparaciones y extraer algunas conclusiones.

"Me esfuerzo por conducir lo divino que hay en mí a lo divino que hay en el universo", cuenta Porfirio que fueron las palabras de Plotino en el momento de su muerte (Vita, 2, 26-27). A lo largo de las Enéadas es recurrente este tema de la conversión del alma, de su retorno al principio y origen. Pero esta preocupación por la émı más íntimo a través del conocimiento y de la práctica de la virtud, está en estrecha conexión e interdependencia con el otro gran tema recurrente en la

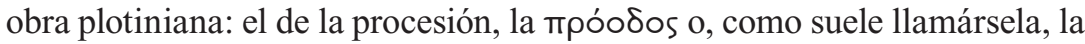

${ }^{2}$ Gerson, L., Plotinus, London and New York, Routledge,1994, p. XVII.

${ }^{3} \mathrm{Cf}$. las observaciones de H. Oosthout, Modes of Knowledge and the Transcendental. An Introduction to Plotinus Ennead 5.3 [49], Amsterdam-Philadelphia, B.R. Grüner, 1991, p. 4-10.

${ }^{4}$ De este aspecto me he ocupado en "Plotin faca à Platon. Un exemple d'exégèse plotinienne (Ennéade VI 8 [39], 18)", en FATAL, M. (ed.), Études sur Plotin, Paris, L'Harmattan, 2000, p. 193-216. 
emanación. En efecto, Plotino dedica buena parte de sus escritos a explicar la naturaleza y la estructura de la realidad en su conjunto. Reflexión moral y reflexión metafísica son en Plotino las dos caras de la misma moneda.

La lectura de las Enéadas permite advertir que Plotino quiere que sus lectores comprendan su modo de concebir la estructura de la realidad y, a la vez, la naturaleza íntima del hombre y que para lograrlo se vale de dos procedimientos diferentes o, podríamos decir, de dos aspectos complementarios de un mismo método, ambos filosóficos más que místicos o religiosos ${ }^{5}$ : uno es el movimiento de procesión o de descenso que lleva a cabo la realidad a partir de la unidad; el otro es la descripción del ascenso del alma, de su conversión purificadora hacia su fuente. Ambos aspectos se hallan a menudo tan imbricados que no es siempre fácil poder separarlos en los textos; y ello se comprende, en la medida en que el camino de ascenso del alma consiste en cierto modo en rehacer en sentido inverso el proceso que la realidad en su conjunto cumple al proceder.

Plotino concibe la realidad como un dinamismo de naturaleza espiritual. La realidad es una continua producción de lo inferior por lo superior, producción que se verifica mediante la contemplación. Más aun, la contemplación, la $\theta \varepsilon \omega p$ pía, es producción, es moínor $5^{6}$. Todo cuanto existe tiene un punto de partida, un primer productor que, paradójicamente produce sin producir, permaneciendo en sí mismo. Es unidad y unicidad absoluta, más allá de toda dualidad. Cuanto surge de él surge sin el que él lo quiera, ni se incline, ni se lo proponga. La perfección misma que le es propia hace que algo surja de él, como un desborde. El primer producto nacido de lo Uno, en dos etapas (procesión y conversión), es la Inteligencia, primer grado o nivel en que se estructura la realidad después de lo Uno. De la autocontemplación de la Inteligencia procede el alma, segundo grado o nivel en que se estructura, de modo más múltiple y complejo, la realidad. Y el alma, a su vez, produce como imagen de sí misma las formas que estarán en lo sensible y produce, además, como "soporte" de esas formas la materia, último escalón de la realidad, carente ya de vida y de capacidad productora, carente de pensamiento, "cadáver adornado", oscuridad, impasibilidad. Como la realidad es de naturaleza espiritual, cada nivel ontológico es simultáneamente un nivel en que el pensamiento o el conocimiento se estructuran de determinadas maneras, cada vez más múltiples, más complejas y cada vez más imperfectas. Así pues, cada nivel ontológico es asimismo un nivel noético o teorético.

A este movimiento de despliegue le corresponde aquel otro, el de repliegue, que debe cumplir el alma humana, la gran viajera, encargada de retornar

${ }^{5}$ Cf. Oosthout, H., ob. cit., p. 6.

${ }^{6} \mathrm{Cf}$. especialmente el tratado III 8.

Classica, Belo Horizonte, v. I9, n. I, p. 59-73, 2006 
a su fuente, mediante una progresiva simplificación y unificación. La estructura propia del universo como un todo es propia también del ser humano, que contiene en sí los mismos niveles ontológicos y noéticos. No debe olvidarse que para Plotino el alma humana, que posee la misma naturaleza que la de la realidad en su conjunto, aunque inteligible es intermediaria, una suerte de puente tendido entre lo inteligible y lo sensible y que su "conversión", su غ̇тıотрофń, es a la vez cognoscitiva y moral, teórica y práctica.

He recordado a grandes trazos la doctrina de las hipóstasis para poner en contexto el tratamiento que de los diferentes modos de conocimiento nos ofrece Plotino. No es mi intención hacer un estudio exhaustivo. Me limitaré acá a establecer la diferencia entre tres modos de conocimiento, de pensamiento o de aprehensión que se verifican en los tres niveles diferentes de la realidad y del alma humana. Llamaré nivel supranoético al que corresponde a lo Uno, Tò Év, nivel noético, al de la inteligencia, voũs, y nivel dianoético, al del alma, $\Psi \cup \times$ ń. Comenzaré encarando, por motivos que ofreceré más adelante, el nivel noético y el modo de conocimiento que le es propio. Pasaré luego al nivel dianoético y dejaré para el final el nivel supranoético. Haré luego algunas breves consideraciones finales.

\section{El nivel noético}

Conviene aclarar de entrada que para Plotino conocer es una noción más amplia que pensar, porque la percepción o la opinión son también modos de conocer, pero pensar es conocer. Resulta muy difícil, si no imposible, trazar en los textos una distinción entre el vocabulario del voєĩv y el del

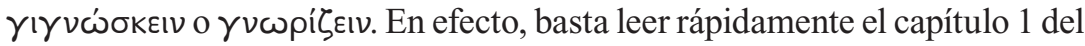
tratato $\mathrm{V} 3^{7}$, en cuyo título aparece $\gamma \nu \omega$ pí $\varepsilon \mathrm{v}$, para advertir que comienza

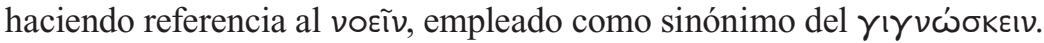

La inteligencia, voũs, representa la aparición de la primera multiplicidad, pero de una multiplicidad que es unidad. Ella es "lo uno-que-es" (y Plotino retoma acá la segunda hipótesis del Parménides de Platón), es “unomúltiple" (V 3,15,11; V 4,1,21; V 1,8,26). Es primer pensamiento, primera vida y primer ser, tríada cuya fuente es sin duda el Sofista de Platón. "El ser es pleno cuando asume la forma del pensar y del vivir. Pensar, vivir y ser se dan entonces simultáneamente en lo que es. Si algo es, también es inteli-

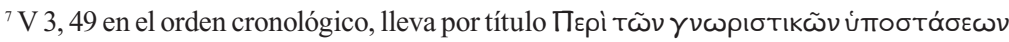

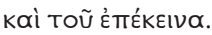

${ }^{8}$ Cf. el artículo ya clásico de НAdot, P. "Être, Vie, Pensée chez Plotin et avant Plotin", en Sources de Plotin, Entretiens sur 1'Antiquité Classique, 5, Vandoeuvres-Genève, 21-29 août 1957, Fondation Hardt, 1960, p. 107-157. 
gencia, y, si es inteligencia, también es: pensar y ser se dan conjuntamente. Múltiple y no uno es, en consecuencia, el pensar; a cuanto no es múltiple no debe corresponderle el pensar" (V 6,6,20-25). La actividad de la inteligencia consiste en pensar, voeĩv, y el objeto de su pensar es ella misma. "La facultad de pensar (Tò фроveĩv) no es en ella un añadido; si piensa (voعĩ) algo piensa por sí misma y si posee algo lo posee por sí misma. Pero si piensa por sí misma y desde sí misma, ella es lo que ella piensa...ella piensa los seres (Tà övta) y los hace existir; ella es, pues, los seres. Tendrá, entonces, que pensarlos como algo que se halla en alguna otra parte, o bien como algo que está en ella misma y por ende idénticos a ella. Que estén en alguna otra parte es a las claras imposible. En efecto, ¿dónde podrían estar? Tendrá pues que pensarlos como idénticos a ella y en ella misma" (V 9,5,4-16). Y esos övтa constitutivos de la inteligencia son la pluralidad de formas inteligibles, una suerte de trasposición del mundo platónico de Ideas, podríamos decir.

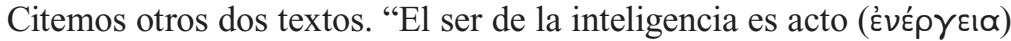
y nada hay hacia lo que ese acto tienda; tiende, pues, hacia sí misma. Al pensarse ( $v \circ \tilde{\omega} v)$ a sí misma, tiende hacia sí misma y ejerce su acto sobre sí misma" (V 3,7,18-20). La inteligencia se piensa a sí misma porque piensa su propio acto.

"Lo que se piensa a sí mismo, en razón de su propio ser, no está separado $<$ de lo pensado $>$, sino que, por estar unido a sí mismo, se ve a sí mismo. Ambos términos resultan, en consecuencia, una unidad. Lo que se piensa a sí mismo piensa en mayor grado porque posee lo que piensa y piensa de un modo primario porque lo pensante debe ser tanto unidad como dualidad. $\mathrm{Si}$ no fuesen una unidad, lo pensante y lo pensado serían diferentes (lo pensante no sería entonces pensante en sentido primario, porque si tuviese pensamiento de otra cosa no sería lo primariamente pensante: no poseería como algo propio lo que él piensa y, en consecuencia, no se pensaría a sí mismo. $\mathrm{Si}$, en cambio, posee lo que piensa como algo propio y piensa, entonces, en modo primordial, ambos términos serán una unidad; en consecuencia, ambos deben ser una unidad). Si, en cambio, lo pensante y lo pensado fuesen una unidad, pero no fuesen, además, dos términos, lo pensante no poseería lo que él piensa y, en consecuencia, tampoco sería pensante. Simple y no simple ha de ser, entonces, lo que se piensa a sí mismo" (V 6,1,4-14). La inteligencia "es dos porque piensa, pero porque se piensa a sí misma es uno" (V 6,1,24).

En el tratado V $3^{9}$ Plotino afirma una y otra vez que conocimiento en

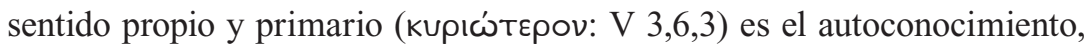

\footnotetext{
${ }^{9}$ Además del libro de Oosthout antes citado, señalo dos importantes y detallados comentarios a este tratado: BeIERWALtes, W., 'Selbserkenntnis': Selbsterkenntnis und Erfahrung der Einheit. Plotin Enneade V, 3. Text, Úbersetzung, Interpretation, Erläuterungen, Frakfurt/Main,
} 
dado que éste no requiere de nada exterior a sí mismo (V 6,1,4-11; V 3,13,1216). Pensamiento de sí mismo es pensar en sentido primario: Kupíws દ̇ఠтi vocĩv $(\mathrm{V} 3,13,14)$. La inteligencia posee el autoconocimiento y Plotino insiste en la identificación entre la inteligencia y el inteligible, entre voũs y vontóv, pero, además, entre la inteligencia y su acto, entre voũs y vónoıs. Ser, objeto de intelección, inteligencia y acto intelectual no son sino uno. "Para pensar, debe haber una inteligencia que piensa, además, esta inteligencia debe tener un objeto inteligible (vontóv) y, en fin, si se trata del ser pensante en sentido primario, debe tener ese objeto inteligible en sí misma" (V 6,2,2-5).

Esta idea del pensamiento que se piensa a sí mismo, así como aquella otra de la interioridad de lo objetos inteligibles en la inteligencia, tienen clara raíz en la concepción aristotélica del primer motor inmóvil, puro pensa-

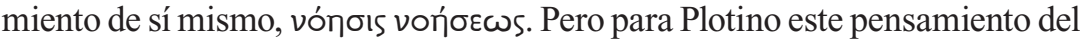
pensamiento no puede ocupar el primer rango en la realidad, porque aunque inteligencia e inteligible constituyan una unidad, el pensar encierra, por su propia naturaleza una dualidad: lo que piensa y lo pensado. Por encima de la inteligencia está el primer principio que no encierra ninguna duplicidad y que es absoluta unidad.

Ahora bien, decir que la inteligencia se piensa a sí misma es lo mismo que decir que se autocontempla, tal como Plotino insiste en III 8. Este tratado, número 30 en el orden cronológico y uno de los cuatro escritos contra los gnósticos ${ }^{10}$, está dedicado a la noción de contemplación, $\theta \varepsilon \omega$ pía $^{11}$. Allí Plotino recalca el carácter productivo de la contemplación. Hay grados de producción y hay grados de contemplación. No es mi interés detenerme en los detalles de este tratado, donde Plotino comienza presentando el tipo de contemplación que es propio de la naturaleza, de la фúøıs, para ir elevándose de grado en grado hasta llegar al principio supremo, lo Uno.

El acto de pensar es un acto de contemplación y la de la inteligencia es la primera de las contemplaciones de esa cadena de contemplaciones que

Klostermann, 1991 (hay trad. italiana) y, más reciente, HAM, B., Traité 49 (V, 3). Les Écrits de Plotin publiés dans l'ordre chronologique, intr., trad., comm. et notes, sous la dir. de P. Hadot, Paris, Éd. du Cerf, 2000. Remito también a los trabajos compilados por MoniQue Dixsaut en La connaissance de soi. Études sur le traité 49 de Plotin, Paris, Vrin, 2002. Para el plan, la estructura y temas del tratado, ver los artículos, incluidos en ese volumen, de C. D' Ancona Costa, "Plan du traité" (p. 173-177) y de L. Lavaud, "Structure et thèmes du traité 49" (p. 179-207).

${ }^{10}$ Cf. Cilento, V., Paideia antignostica. Ricostruzione d'un unico scritto da Enneadi III 8, V 8, V 5, II 9, Biblioteca nazionale, Serie di classici greci e latini. Testi con commento filologico, 9, Firenze, Le Monnier, 1971.

${ }^{11}$ Sobre la contemplación, cf. Cilento, V., "Contemplazione", en Saggi su Plotino, Milano, U. Murcia \& Co., 1973, p. 5-27; Eвorowicz, W., "La contemplation selon Plotin", Giornale di metafísica 12, 4 (1957) 472-518 y 13, 1 (1958) 42-82; Hunt, D., "Contemplation and hypostatic procesión in Plotinus", Apeiron 15, 2 (1981) 71-79. 
producen todo el despliegue de lo real. La unidad de sujeto contemplante y objeto de contemplación no es resultado de una "apropiación" del objeto llevada a cabo por el sujeto contemplante, sino que es una unidad "esencial" o "sustancial" (oúoíá), pues "es lo mismo ser y pensar", dice Plotino citando el fragmento 3 de Parménides (III 8,8,7-8). Para verse y conocerse a sí misma, la inteligencia no debe salir de sí, porque ejerce la contemplación de un objeto que coincide con ella, un objeto que le está inmediatamente presente. Su visión es, pues, directa e "intuitiva". No debe llevar a cabo ningún tipo de "procedimiento" intelectual para conocerse a sí misma. No

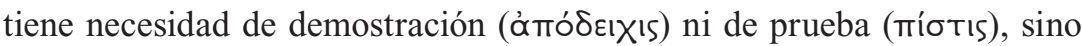

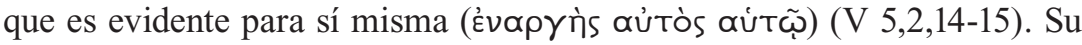
conocimiento es su conversión (غ̇ாıтрофń) hacia sí misma (V 3,6,40ss), el pensar que le es propio consiste en una visión noético-intuitiva de sí misma. En verdad, la inteligencia aspira a pensar lo Uno, pero no logra pensarlo en sí mismo y lo que ella piensa es lo Uno traspuesto, espejado en ella. "Es así que esta inteligencia múltiple, cuando intenta pensar lo que está más allá, lo piensa como una unidad; pero aunque quiere apresarlo en su simplicidad, acaba siempre por recibir en sí misma algo diferente que se ha pluralizado: se dirigió hacia él no como inteligencia sino como una visión que aún no veía, y esa visión acabó poseyendo lo que ella misma pluralizó" (V 3,11,1-5). La inteligencia multiplica lo uno y la conversión hacia lo Uno acaba siendo una autoconversión. Sólo puede aprehender a lo Uno en sí misma, precisamente porque es inteligencia y no absoluta simplicidad.

\section{El nivel dianoético}

El alma, aunque de naturaleza plenamente inteligible (IV 1,1-3; IV 7, $8^{5}, 46-47$; III 6,6,1; etc), es la intermediaria o el puente entre lo sensible y lo inteligible (IV 6,3,10; IV 3,12,30-32; IV 4,3,10-12; IV 7,13,17-18). Plotino nos dice que tiene una doble vida, que es anfibia (IV 8,4,31-32). El alma posee dos funciones: una superior, vuelta hacia la inteligencia y una inferior, vuelta hacia lo sensible y corpóreo, función ésta que consiste en dar forma y vida a lo inferior a ella. Esta división del alma en dos partes o funciones es un tema que aparece reiteradamente en las Enéadas, enfocado desde diferentes perspectivas. No es fácil entender el modo en que Plotino explica cuál es la relación entre esas dos funciones. Una respuesta, compleja, por cierto, la hallamos en el tratado III 8, en particular en el capítulo 5. Al ocuparse del alma, Plotino establece una distinción entre su parte superior y "racional", que permanece inmóvil contemplando la inteligencia, y su parte inferior, que procede de la primera, y que, apartándose de ella, "desciende": "La parte racional del alma permanece en lo alto cerca de la cima, siempre fecundada 
e iluminada; la otra parte del alma, que participa de la primera gracias a una participación que es la primera por la que un participante participa, esa parte del alma procede. Al avanzar, deja a la primera parte de sí misma, a la más alta, permanecer donde la ha dejado. Ya que si abandonara esta parte anterior ya no estaría en todas partes sino sólo donde acabase su marcha. Pero la parte que procede no es igual a la permanece" (III 8, 5, 10-17). La parte racional

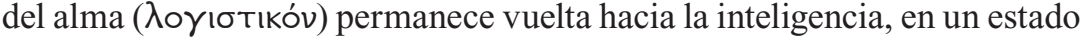
de plenitud e iluminación eternas; la otra parte "participa" de la primera y

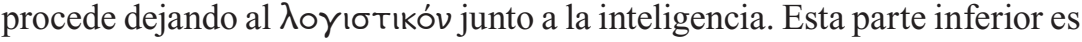

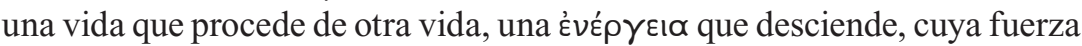
es más débil que la de la parte superior. La parte que desciende y se aleja de la primera es resultado de la contemplación ejercida por la porción superior: es el objeto de contemplación de ésta y ejerce, también ella, una actividad contemplativa de menor intensidad (cf. III 8, 5, 17-30).

En el alma, a diferencia de lo que acontece en el nivel de la inteligencia, sujeto y objeto ya no son esencialmente una unidad. Pero aunque no son una unidad, buscan serlo y, por ello, no son completamente diferentes ni están yuxtapuestos como si se tratara de dos cosas totalmente distintas. Uno y otro son el alma. El alma contemplante no tiene un objeto diferente de ella misma, pero ese objeto no le está inmediatamente presente. El alma debe distenderse, debe desdoblarse para poder aprehender su objeto que es ella misma y eso a

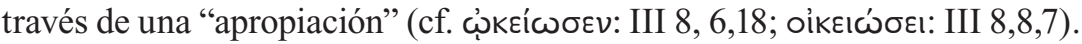
El contenido del alma, su $\lambda$ óyos, no debe ser exterior al alma que conoce,

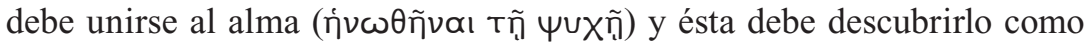
propio (oíkẽ̃ov) (III 8,6,19-20), debe proyectarlo fuera de sí y — metáfora que Plotino emplea - debe ponerlo al alcance de la mano, "manipularlo"

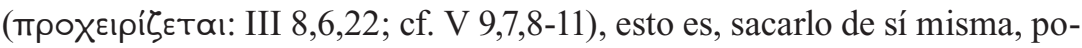
nerlo frente a sí, para poder poseerlo y comprenderlo y comprenderse así a sí misma. Pero el alma, a través de este proceso de exteriorización de su objeto, se vuelve de algún modo diferente de sí misma y lo contempla como si fuera otra cosa y no ella misma. El alma funciona así como una inteligencia, pero como una inteligencia que ve algo diferente de sí misma. La actividad del alma no es, pues, como la de la inteligencia, de carácter noético-intuitivo, es de tipo dianoético, "discursivo". Ella "ve su objeto como algo diferente

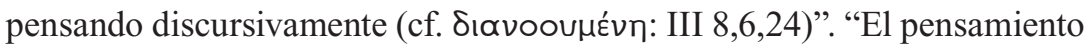
discursivo, para poder expresarse, capta las cosas unas después de otras; él

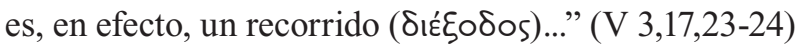

También en otros tratados Plotino insiste en la diferencia entre el pensamiento propio del alma y el de la inteligencia. En I 2,3,24ss, por ejemplo, nos dice que el alma también goza de la capacidad noética, que ejerce una actividad de pensamiento, pero diferente de la que es propia de la inteli- 
gencia, porque la actividad pensante del alma es secundaria y derivada de aquella de la inteligencia y funciona de modo diferente.

En V 3, uno de los últimos tratados de Plotino, complejo y difícil, el abordaje se hace desde la perspectiva del alma humana. El tratado, denominado por Porfirio "De las tres hipóstasis que conocen"12 está consagrado al complejo problema de la posibilidad del autoconocimiento. El tratado analiza en un camino de "ascenso" la posibilidad de autoconocimiento del alma, de la inteligencia y de lo Uno. Desde el capítulo 2 Plotino examina la

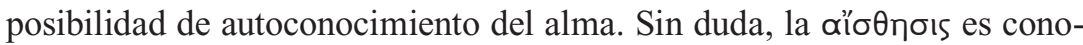
cimiento de un objeto exterior, aun cuando se trate de la percepción de lo que acontece dentro del propio cuerpo, y otro tanto sucede con la $\delta o ́ \xi \alpha$. En

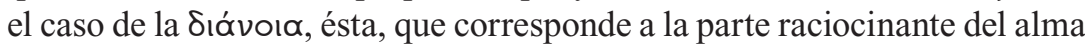

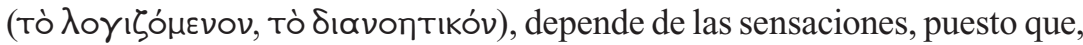
uniendo y dividiendo ${ }^{13}$, juzga a partir de imágenes ( $\left.ф \alpha \nu \tau \alpha ́ \sigma \mu \alpha \tau \alpha\right)$ derivadas de la sensación. Ella entonces es tributaria de la exterioridad y, en ese sentido, conoce algo que le es extraño, exterior y no a sí misma. Pero, por otra parte, la Sıớvoı tiene relación también con el voũs. Ella en realidad es intermediaria entre interioridad y exterioridad.

Como antes señalamos, conocimiento en sentido propio y primario (кupı́́tєpov: V 3,6,3) es el autoconocimiento, dado que éste no requiere de nada exterior a sí mismo (V 6,1,4-11; V 3,13,12-16). Pensamiento de sí mismo

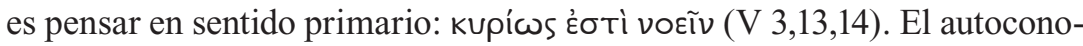
cimento va asociado siempre a la noción de autoconversión, de غ̇mı Eis Ėautó (cf., por ej., V 3,2,15-16).

Si la vónoıs es de algo exterior, es deficiente y ya no es voeĩv en sentido

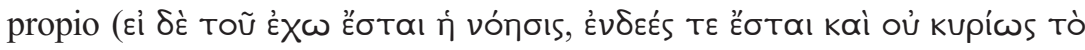
ขовĩv: 15-16). Sin embargo, Plotino parece reconocer un sentido secundario y derivado del autoconocimiento: el del alma (V 3,4,23 y 6,2-3). El alma se piensa a sí misma en tanto depende de la inteligencia ${ }^{14}$. Así, puede decirse que en un sentido el alma se autoconoce y que ella, en otro sentido, no se autoconoce. Ya que sólo se conoce o se piensa a sí misma en tanto, por su parte superior, se hace inteligencia o participa de su actividad. El alma tiene como modo propio el razonar, el $\lambda \circ \gamma^{\prime} \zeta \zeta_{\varepsilon \sigma \tau \alpha l .}$ Y lo propio de éste es conocer o pensar algo exterior y no a sí mismo.

${ }^{12}$ Sobre este tratado, cf. nota 8.

${ }^{13}$ Repárese en la resonancia platónica de estos dos términos, usados tanto en Fedro como en Sofista y Político para señalar las dos operaciones del proceder dialéctico. También Plotino en el tratado I 3 sobre la dialéctica retoma tal tema.

${ }^{14}$ Sobre esta cuestión, ver KüHN, W., "Comment il ne faut pas expliquer la connaissance de soi-même", en Dixsaut. M. (ed.), ob. cit., p. 229-266. Cf. p. 237 y la crítica de Kühn a Gerson. Kühn subraya acertadasmnte la importancia del oútos en V 3, 4, 23. 


\section{El nivel supranoético}

Pasemos ahora al plano de lo Uno, del primer principio absolutamente simple, sin repliegues, sin fisuras. Como veremos, Plotino enfrenta acá un problema, en la medida en que se ve obligado a mostrar que la máxima perfección que le es propia excluye perfecciones como la del pensamiento o del conocimiento ${ }^{15}$.

En la mayoría de los textos en los que Plotino describe la generación de la Inteligencia a partir de lo Uno, insiste en el hecho de que toda generación exige la permanencia en sí mismo del generador, especialmente en el caso de lo Uno. En efecto, “...lo que nace de lo Uno nace de él sin que él se mueva... Puesto que él es inmóvil, si después de él nace algo en segundo término, éste debe comenzar a existir sin que lo Uno se mueva, sin que hacia allí se incline, sin que lo desee, y, en una palabra, sin ningún movimiento" (V 1, 6, 22-27). Lo Uno permanece inmóvil, pues todo movimiento es una aspiración y lo Uno, él, no aspira a nada, porque está en la cima (III 9, 9, 1-5).

Lo Uno es lo que se basta totalmente a sí mismo, no busca nada, está

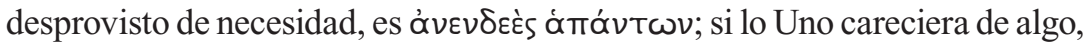
ya no sería uno (VI 9, 6, 16-42).

Lo Uno está más allá del ser, más allá del pensamiento, más allá de la vida, una maravilla que no tiene en sí ni ser ni pensamiento; solitario en sí mismo, no tiene ninguna necesidad de las cosas que vienen después de él (VI 7, 40, 28-32). Al estar solo, más allá de todo, lo Uno no piensa. Si hubiera en él pensamiento, ya no sería uno, sino múltiple, porque la actividad de pensar comporta siempre una alteridad y una dualidad (Hay un buen número de pasajes donde Plotino hace tales afirmaciones. Por ejemplo, V 3, 10, 20ss; V 6, 2, 17ss; V 6, 3, 20ss; V 6, 4, 1ss; V 6, 6, 23ss; V 1, 4, 25ss). En V 3, 10, escribe: "es necesario entonces que lo que piensa, puesto que piensa, consista en dos términos; que uno de estos dos términos sea exterior a él, o que los dos sean lo mismo, el pensamiento trata siempre sobre una diferencia (y necesariamente también sobre una identidad)"'16 (V 3, 10, 23-25). V 6, 2, 14-20:

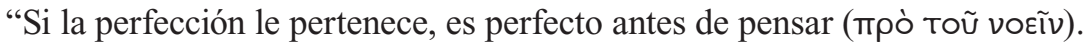
No tiene necesidad del acto de pensar, porque se basta a sí mismo antes del

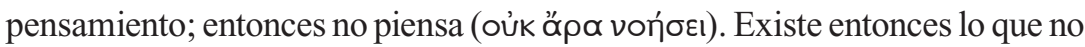
piensa, lo que piensa primariamente, y lo que piensa en sentido secundario. Además, si lo primero pensara, alguna cosa le pertenecería, no sería entonces

\footnotetext{
${ }^{15}$ Sobre este aspecto, retomo acá abreviadamente mi trabajo "L'Un est-il intelligible?", en Dixsaut, M. (ed.), ob. cit., p. 73-90.

${ }^{16}$ He aquí dos de los cinco géneros primeros que Plotino toma del Sofista de Platón para trasponerlos a la Inteligencia.
} 
primero, sino segundo; no sería uno, sino múltiple; sería todo lo que piensa. Y aunque se pensase sólo a sí mismo [lo Uno H-S] sería múltiple"17.

Asimismo, en V 1, 9, en el curso de la crítica a la concepción aristotélica de los motores inmóviles, dice Plotino: "Aristóteles afirma que el primero es separado e inteligible, pero desde el momento en que dice que él se piensa a sí mismo destruye su carácter de primero" (7-9).

Lo Uno, entonces, no puede pensar, no puede voeĩv. Él no piensa en otra cosa y no se piensa tampoco a sí mismo. Si él se pensara - argumenta Plotino - , debería ser ignorante de sí mismo antes de pensarse, y tendría necesidad del pensamiento para conocerse a sí mismo, lo que es absurdo (VI 9, 6, 42ss).

No tiene vónoıs; pero no hay tampoco en él ni percepción, ni conocimiento de sí mismo ni conciencia de sí mismo (III 9, 9, 14; V 3, 13, 6). Percepción, conocimiento, conciencia, he aquí actividades que comportan siempre una dualidad sujeto-objeto, dualidad que lo Uno no puede acoger en su unidad absoluta, sin ninguna diversificación.

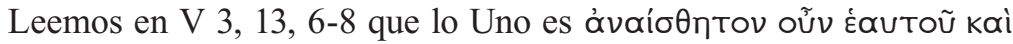

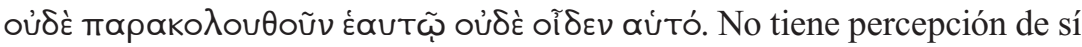
mismo, no se comprende ni se conoce a sí mismo. Debe excluirse de lo Uno

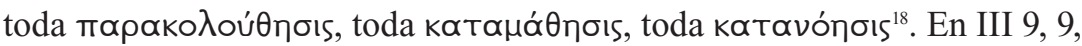

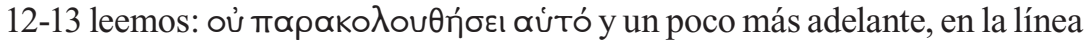

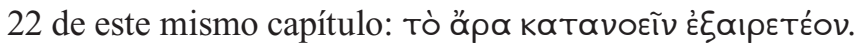

Plotino, sin embargo, tiende a remarcar que el hecho de que en lo Uno no haya conocimiento no quiere decir que haya en él ignorancia, porque ella misma, al igual que el conocimiento, encierra una alteridad, cuando una cosa ignora a otra. De forma análoga, el hecho de que lo Uno no piense no implica un defecto, no implica de ningún modo que él sea no inteligente.

${ }^{17} \mathrm{Cf}$. V 6, 3, 20-23: "Si existe una multiplicidad, es necesario, antes de esta multiplicidad, una unidad. Si entonces lo que piensa es una multiplicidad, es necesario que el pensamiento no esté en lo que no es una multiplicidad. Y tal es lo Primero. La facultad de pensar y la inteligencia están entonces en los seres posteriores a él". Cf. V 6, 4, 1-7: Además, si el Bien debe ser simple y sin necesidades, aquello de lo que no necesita no le está presente. Y además, nada absolutamente está presente en él; la facultad de pensar no está en él entonces presente. No tiene necesidad de pensar. Además, no piensa nada, porque no tiene otra cosa que pensar. En III 9, 7, Plotino le niega a lo Uno una vez más la vónoıs: a diferencia de la inteligencia que tiene la vónoıs dirigida hacia otra cosa, "lo Uno no tiene pensamiento, porque lo que tiene el pensamiento es necesariamente doble y deficiente, aunque se piense a sí mismo (1-6). Y en III 9, 9, 1: lo primero, que está más allá del ser, no piensa.

${ }^{18}$ Para un análisis del significado de estos términos, ver Schwyzer, H.R., "Bewusst und Unbewusst bei Plotin", en Sources de Plotin, ob. cit., p.341-390; WARren, E., "Consciousness in Plotinus", Phronesis 9 (1964) 83-97; Violette, "Les formes de la conscience chez Plotin", Revue des Études Grecques 107 (1994) 222-237. 
En VI 8, 16, 15-16, Plotino dice además que lo Uno es una suerte de inteligencia: oĩov voũs; que lo Uno, por así decirlo, mira hacia sí mismo (oĩov

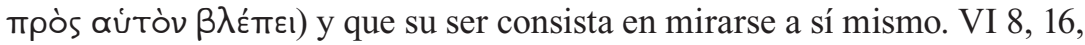
$20 ; 18,21$ : en lo Uno hay un voũs que no es voũs. También dice que él es la inteligencia interior (ó Ěvઠov voũs: V 3, 14,13). "Cuando nosotros alcanzamos la inteligencia pura y usamos de ella, vemos que es la inteligencia interior, la que ha hecho don a la inteligencia del ser y de las otras cosas..." (11, 13-15). No es un sujeto pensante, no es voũv, sino que es en efecto vónoıs, aunque vónбıs oủ vorĩ, un pensamiento que no piensa, sino que es la causa de que otra cosa pueda pensar (VI 9, 6, 54-56). VI 9, 6, 52-55: "pues no hay que concebirlo según la categoría [ubicarlo en la categoría de] de lo pensante

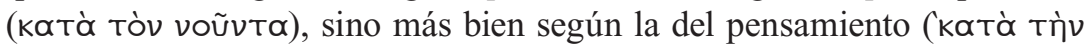
vónбıv); ahora bien, el pensamiento no piensa (cf. VI 7, 37, 16), sino que es

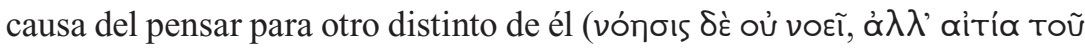
vocĩv ở $\lambda \lambda \circ$ ), y la causa no es idéntica a lo que es causado".

Por otra parte, que lo Uno no tenga conciencia de sí mismo no quiere decir que sea completamente "inconsciente". Lo Uno se posee a sí mismo (III 9, 9, 5-6). "[Lo Uno] no está, por así decir, privado de conciencia (oúk

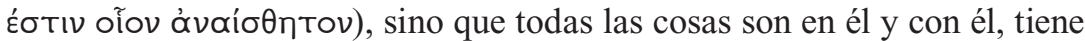

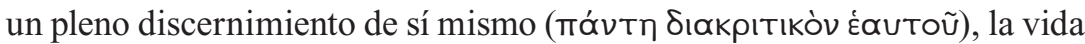
está en él y en él están todas las cosas y su pensamiento (katavónoıs), que es él mismo, por una suerte de conciencia (oíovei бuvaı un reposo eterno y en un pensamiento que actúa de una manera diferente al pensamiento de la inteligencia" (V 4, 2, 16-20).

Este pasaje no parece estar de acuerdo con los otros a los que hemos hecho referencia. En efecto, ahora leemos que lo Uno no está desprovisto de conciencia, sino que, por el contrario, la razón del nacimiento de la inteligencia a partir de lo Uno se encuentra en el hecho de que lo Uno tiene algún tipo de "percepción" de sí mismo, de "conciencia", de vida y de pensamiento, aunque de un tipo diferente de aquellos que la inteligencia posee. Debe no obstante subrayarse que Plotino atribuye la ouvaío $\theta$ noıs a lo Uno no sin res-

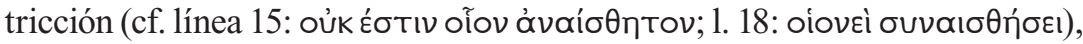
e igualmente la vónoıs (cf. 11. 18-19). Lo Uno posee entonces algo similar a

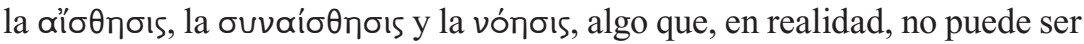
designado como tal más que traslaticiamente o de modo aproximativo.

Lo posesión que lo Uno tiene de sí mismo, o el ser solo consigo mismo, el discernimiento que él tiene de sí mismo consisten en una simple intuición,

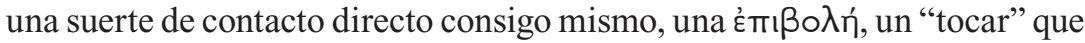
coincide consigo mismo, dado que no existe ninguna distancia ni diferencia respecto de sí mismo (VI 7, 39, 1-4). Se podría decir que lo Uno coincide con el acto de "autopercepción", de aprehensión de sí mismo. Es imposible 
establecer en lo Uno una diferenciación entre voũv y vontóv. Y es en el

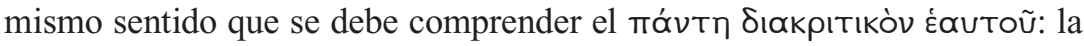
Sıákpıøıs que lo Uno tiene de sí mismo coincide consigo mismo, puesto que todo es en él.

\section{Conclusiones}

En lo anterior he tratado de perfilar los tres diferentes modos de conocimiento que según Plotino son los propios de la inteligencia, del alma y de lo Uno. Me interesa ahora, para terminar y recapitular, subrayar que el punto nodal, que le sirve a Plotino para articular los diferentes modos de conocimiento, es el modo de conocimiento propio de la inteligencia, que es, como dijimos, el pensamiento por excelencia, el pensamiento en sentido genuino, el pensamiento "intuitivo" de sí mismo. Este tipo de pensamiento que revierte sobre sí mismo, requiere unidad sujeto-objeto e interioridad. A diferencia de lo que ocurre en el alma, la inteligencia no se extraña, sino que permanece en sí. Ella es primera dualidad y encierra entonces una distinción entre sujeto y objeto, pero esa distinción no implica distancia ni alteridad entre ellos. En la inteligencia, pensar o conocer, por su carácter de interioridad e inmediatez,

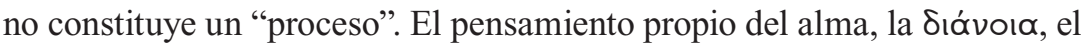
raciocinio o la discursividad, supone en cambio una exteriorización, un salir fuera de sí como condición para reapropiarse de sí mismo. El alma, a diferencia de la inteligencia, se extraña, enfrenta a su objeto que es ella misma y puede conocer a ese objeto como objeto pero, además, al reapropiarse de él, que es ella misma, se conoce a sí misma, aunque de un modo diferente al modo en que la inteligencia se conoce a sí misma. El conocimiento propio del alma exige una reapropiación de lo que se ha exteriorizado, un dis-curso, un recorrido, un "proceso", y en tal sentido el alma no posee un conocimiento de sí al modo en que lo posee la inteligencia, pero, sin embargo, en sentido restringido, secundario y derivado puede hablarse de un autoconocimiento. Cuando se trata del primer principio, que por su absoluta simplicidad debe escapar al pensamiento, y aun al ser y a la vida, Plotino proyecta en él, a partir del autoconocimiento que se verifica en el nivel de la inteligencia, algo que está más allá del autoconocimiento, pero que es como un autoconocimiento, algún tipo de autopercepción, de relación consigo mismo, porque lo más perfecto no puede ser inerte. En este plano, no sólo no hay alteridad ni distancia entre sujeto y objeto, sino que no hay tampoco distinción entre ellos. Y he subrayado el como (oĩov) intencionalmente. Plotino, en efecto, recurre a él en muchas ocasiones para poder hablar de lo Uno de modo impropio y restrictivo, particularmente en la Enéada VI 8, sobre la libertad y la voluntad de lo Uno, partiendo de propiedades de lo que es posterior a él. 
Pero no puedo detenerme ahora en este punto. Dicho de otro modo, si el conocimiento de sí propio y genuino es el de la inteligencia, el del alma es un autoconocimiento producto de una exteriorización y reapropiación de sí y, en consecuencia, en sentido derivado, y el de lo Uno es un autoconocimiento en sentido traslaticio, metafórico, restrictivo o, si se quiere, eminencial. Podría decirse, creo, que en la inteligencia se verifica el autoconocimiento, que es verdadero conocimiento, que en el alma se da un autoconocimiento de segundo rango, al que podríamos denominar "infra-autoconocimiento" y que en el nivel de lo Uno cabría hablar de un "supra-autoconocimiento". Como antes señalé, me parece que, en tal sentido, el punto de referencia y articulación es el modo de conocimiento de la inteligencia. La inteligencia, en efecto, es una imagen pluralizada de lo Uno, así como el alma es la imagen aún más pluralizada de la inteligencia. Y como Plotino, fiel platónico, no puede abandonar el principio de causalidad según el cual la causa debe poseer de algún modo las propiedades que se hallan en su efecto, le resulta altamente problemático no acordarle a lo Uno, que es causa productora del pensar, algún tipo de pensar. Lo Uno puede llamarse pensamiento en tanto fuente del pensar, en tanto causa del pensar. Y otro tanto ocurre con la vida y con el ser. Pero esto nos llevaría a incursionar en otro tema recurrente en las Enéadas: la inefabilidad de lo Uno y el modo de acceso a él, que sería tema de otro tratamiento que no puedo emprender aquí.

RESUMEN. Tras una beve introducción destinada a recordar las grandes líneas del sistema plotiniano, el trabajo analiza tres modos de conocimiento o de aprehensión que caracterizan a los tres niveles de la realidad o hipóstasis. En el nivel de la inteligencia, noûs, al que se denomina nivel noético, se verifica un tipo de conocimiento genuino, que es el autoconocimiento. En el nivel del alma, psyché, nivel dianoético, se da un conocimiento de tipo discursivo que sólo en sentido secundario y derivado es conocimiento de sí. Por fin, en el nivel de lo Uno, tò hén, nivel supranoético, puede hablarse de un conocimiento de sí sólo en sentido traslaticio y "eminencial", puesto que el primer principio de la realidad, que es absoluta unidad, al no tener duplicidad alguna, en sentido estricto no puede tener pensamiento, ni siquiera de sí mismo, pero, al mismo tiempo, no es algo inerte, sino absoluta plenitud. El trabajo intenta mostrar cómo Plotino se apoya en el tipo de conocimiento de la inteligencia, que le sirve de punto nodal, para articular los tres modos de conocimiento y para caracterizar un "infra-autoconocimiento", el del alma, y un "supra-autoconocimiento", el de lo Uno.

Palabras Clave. Plotino; modos de conocimiento; Uno; inteligencia; alma; autoconocimiento. 
Résumé. Modes de connaissance chez Plotin. Après avoir rappelé les grandes lignes du système plotinien, l'étude analyse trois modes de connaissance qui caractérisent les trois niveaux du réel ou hypostases. Dans l'intelligence, noûs, nomée niveau noétique, il y a un type de connaissance autentique, qui est la connaissance de soi. Dans l'âme, psyché, niveau dianoétique, il y a un type de connaissance discursive, qui n'est connaissance de soi que dans un sens secondaire et dérivé. Dans 1'Un, tò hén, niveau supranoétique, on ne peut parler d'une connaissance de soi que dans un sens translatif et "éminent", car le premier principe du réel, étant unité absolue, n'admet pas de pensée, ni d'autre chose ni de soi même, mais, en même temps, il n'est pas quelque chose d'inerte, mais plénitude absolue. L'étude tente de montrer comment Plotin s'appuie sur le mode de connaissance de l'intelligence, dont il se sert comme point nodal, dans le but d'articuler les trois modes de connaissance et de caractériser une "infra-connaissance de soi", celle de l'âme, et une "supra-connaissance de soi", celle de 1'Un.

Mots-CLÉs. Plotin; modes de connaissance; Un; intelligence; âme; connaissance de soi. 